\title{
Using ArchiMate and TOGAF to Understand the Enterprise Architecture and ITIL Relationship
}

\author{
Marco Vicente ${ }^{1}$, Nelson Gama ${ }^{1,2}$, and Miguel Mira da Silva ${ }^{1}$ \\ 1 Instituto Superior Tecnico, Av Rovisco Pais, 1049-001 Lisboa, Portugal \\ 2 CINAV-PT Navy Research Center, Escola Naval, Alfeite, 2810-001 Almada \\ \{marco.vicente, nelsongama, mms\}@ist.utl.pt
}

\begin{abstract}
Business/IT alignment has become one of the most relevant concerns on organizations. Enterprise Architecture (EA) and ITIL, two distinct governance approaches with different perspectives, have become recently dominant between practitioners. However, parallel EA and ITIL projects can lead to wasted resources and a duplication of costs and efforts. In this paper we propose an EA and ITIL integration using ArchiMate as a common frame of reference. We also want to point out that implementing ITIL is like implementing any other architecture change and demonstrate it by using TOGAF to perform an ITIL implementation on ArchiSurance, a fictitious organization from the well known ArchiMate case study.
\end{abstract}

Keywords: Enterprise Architecture, ITIL, IT Service Management, business/IT alignment, TOGAF.

\section{Introduction}

In the last decades, IT has evolved from its traditional orientation of administrative support to a strategic role, turning business/IT alignment into a major concern. In the early nineties, Henderson [1] proposed a strategic alignment model based on two building blocks: strategic fit and functional integration, using business strategy as the driver and IT as the enabler. This model presented several perspectives on how to integrate business and IT domains, using concepts like information systems service organizations and IT governance [1].

Recently, the growing demand on IT led to the improvement of the key concepts related to IT Governance, namely the ones connected to IT alignment with strategic objectives and cost reduction initiatives [2]. From these Governance initiatives, two main approaches have had major relevance: Enterprise Architecture (EA) and IT Service Management (ITSM).

EA is a coherent whole of principles, methods, and models that are used in the design and realization of an enterprise's organizational structure, business processes, information systems, and infrastructure [3]. Therefore, according to EA approaches, organizations usually share several architectures: business, processes, information, application and technology infrastructure [3415].

X. Franch and P. Soffer (Eds.): CAiSE 2013 Workshops, LNBIP 148, pp. 134-145, 2013.

(C) Springer-Verlag Berlin Heidelberg 2013 
ITSM evolved naturally as services became underpinned in time by the developing technology. In its early years, IT was mainly focused on application development, but as time went by, new technologies meant concentrating on delivering the created applications as a part of a larger service offering, supporting the business itself [6]. ITIL [7] is the de facto standard for implementing ITSM [8. It is a practical, no-nonsense approach to the identification, planning, delivery and support of IT services to the business [9].

Unfortunately, having two different frameworks to approach governance can led to several setbacks. In a time when organizations strive to be efficient and effective, it seems counterintuitive to be wasting resources by having different organizational departments or teams handling both approaches independently.

This paper is part of a wider effort to join both approaches by establishing a specific enterprise architecture for organizations that need to manage IT services. EAs don't focus on specific issues because their goal is to be able to represent every organization. On the contrary, our goal is to narrow it down, and restrict the architecture to organizations that have the management of IT services as an architectural driver, using ITIL principles, methods, processes and concepts to perform IT service management, and general EA principles, methods and models to the design and realization of the remaining organizational structure.

Thus, this paper's goal is to show how ITIL can be approached from an EA point of view, and how this can be used to design ITSM organizations and implement ITIL through EA methods as if it were any other architectural change.

The methodology applied across this paper is Design Science Research, where we develop and validate a proposal to solve our problem 10. The following sections follow the methodology's steps: "Related Work" covers aims and objectives as the awareness and recognition of a problem from a state of the art review giving us the issues that must be addressed. Later on, "Research Problem", exposes the main problem while offering a tentative idea to how these issues might be addressed. Afterwards, "Proposal" presents a proposal as an attempt to solve the previously described problem. Next, we present a "Demonstration" followed by the "Evaluation" comparing the results with the research questions and to conclude we show our proposal applicability and themes for further work.

\section{Related Work}

Here we will introduce what is Enterprise Architecture, followed by TOGAF an EA framework, and ArchiMate - an EA modeling language. Finally we will address ITIL, a best practices model to IT service management.

\subsection{Enterprise Architecture}

Today, business performance depends on a balanced and integrated design of the enterprise, involving people, their competencies, organizational structures, business processes, IT, finances, products and services, as well as its environment [11. 
EA is a coherent set of principles, involving the design and performance of different architectures. It specifies the components and its relationships, which are used to manage and align assets, people, operations and projects to support business goals and strategies 312, concerning those properties of an enterprise that are necessary and sufficient to meet its essential requirements [11. EA is based on a holistic representation of organizations, on views and the ability to map relationships between artifacts and architectures which usually are 3|4|5: Business, Process, Application, Information, and Technology. The alignment between them allows a coherent blueprint of the organization, which is then used for governance of its processes and systems [13].

\subsection{TOGAF}

The Open Group Architecture Framework (TOGAF) is a framework for developing an EA [4. It was developed and is currently maintained as a standard by The Open Group (TOG). The first version of TOGAF, in 1995, was based on the US Department of Defenses Technical Architecture Framework for Information Management (TAFIM) 4|14. Each version of the standard is developed collaboratively by the members of the TOG Architecture Forum [414].

TOGAF provides the methods and tools for assisting in the acceptance, production, use, and maintenance of an EA [4]. It is one of the leading architecture frameworks worldwide, and in its latest version there is increasing reflection on the use of the architecture and its governance [14. The TOGAF documents focus on EA key concepts and TOGAF Architecture Development Method (ADM), a step by step approach to developing an EA [15].

\subsection{ArchiMate}

The ArchiMate EA modeling language was developed to provide a uniform representation for architecture descriptions [15]16. The goal of the ArchiMate project is to provide domain integration through an architecture language and visualization techniques that picture these domains and their relations, providing the architect with instruments that support and improve the architecture process 25. ArchiMate is now also becoming well known in the international EA community, being today a TOG standard [15.

The domains of business, application and infrastructure are connected by a service orientation paradigm, where each layer exposes functionality in the form of a service to the layer above [16. Besides this, it also distinguishes between active structure, behavior and passive structure elements, having also another distinction between internal and external system view. On top of this, ArchiMate is a formal visual design language, supports different viewpoints for selected stakeholders and is flexible enough to be easily extended [16.

\subsection{ITIL}

ITIL was created by the Central Computer and Telecommunications Agency (CCTA), and was first released to the public in the late eighties [14. ITIL is a 
common-practice model possessing the character of a branch standard 8 . While the first version was mainly based on experience in data centers running big mainframes, in 2000 a revised version (v2) was launched becoming the worldwide de facto standard for IT Service Management 14.

The next version of ITIL (v3) appeared in 2007 and covers the major weaknesses identified in the previous versions, namely being too focused on technology 2. Now, instead of focusing on the service itself, the focus lay on this cycle of life, renewal and decommissioning of services, with a greater business-focused perspective [14. The ITIL Core consists of five publications and each book covers a phase from the Service Lifecycle with various processes which are always described in detail in the book in which they find their key application [17.

\section{Research Problem}

There have been some attempts to integrate EA and ITIL. In fact, Brown and Winter 18 proposed an EA expansion to integrate ITIL v2 and Service Oriented Architectures (SOA), having EA as a pivotal concept with ITIL regarded for IT operations. Nabiollahi 19 provides a service based framework for EA to meet the ITSM requirements of ITIL, suggesting an EA extension to involve service architecture layer from ITIL Service Design [20]. The development of an architecture model for IT services is proposed, making it a service layer for EA.

Thorn [21] addresses the relation between ITIL and TOGAF, regarding EA as a fundamental concept for organizational engineering, in which ITIL is included as a framework to an operation model for IT delivered services. He argues that both frameworks can be used together by mapping them, TOGAF covers the development of EA, and is involved in the products conception lifecycle whereas ITIL ensures the delivery and management of IT services to users [2]21].

In the same note, Sante 14 addresses the fact that the recent versions of ITIL and TOGAF keep converging to integration. In fact, in ITIL V3 references are made to architectural concepts, hitherto only found in publications on architecture. The same, although to a much lesser extent, applies to TOGAF 8.1.1: references are made to IT management 14. The author relates the five ITIL books to TOGAFs ADM cycle, showing there are indeed several similarities, but identifying two main differences: a) developing business architecture is part of the TOGAF framework while the scope of ITIL is limited to developing an effective and efficient IT department, whilst developing business architecture is out of scope in ITIL; and b) running IT operations and delivering actual IT services are within the scope of ITIL, while TOGAF does not cover the development and maintenance of a run time environment, neither the way how services are actually produced and delivered [14.

Thus, EA is regarded as a fundamental concept for organizational engineering, and ITSM is regarded as the dominant operations model sufficiently integrated into the former 22. EA guarantees consistency in building new products or services and addresses business requirements, while ITSM guarantees the consistency of services, through the use of standard processes [22]. 
All these integration attempts tried to answer a real problem that should not be taken lightly. However, while all these approaches seem to come close to an integration, they don't propose a definitive and holistic solution. In fact, Braun's [18] and Thorn's 21] work is limited to ITIL v2, what makes it outdated, Nabiollahi [19] proposes a service architecture as a new architecture layer, but doesn't clarify the architectures relationships. As for Sante's 14 work, although we agree upon the approaches used and the conclusions reached, the result isn't a unique body of knowledge with EA and ITIL, but two different frameworks linked by a mapping. Moreover, none of these approaches provide models or a formal representation for the proposed solutions. In fact, what we are looking for is a holistic solution, following the EA approach but using ITIL best practices to perform IT service management.

Our research question is then how can we contribute to this discussion and how can ITIL be integrated with EA in a way that would allow ITIL and EA teams to collaborate on organizational change.

\section{Proposal}

ITIL is often described as a process-based [6] or a process-oriented [17 framework. Although we realize that most of ITIL contents are about describing best practice processes (and the information they use), we believe that limiting ITIL to these only two domains is one of the factors that makes its integration with EA so difficult. That said, we believe that like EA, we should also look at ITIL as a composition of other architectures, linked by a service oriented approach, where functionality is available to the next layer in the form of services.

Additionally, we also realized that it would be harder to integrate two approaches if they didn't speak exactly the same language, so we needed a uniform representation, a common frame of reference. To represent EA we chose ArchiMate as it offers an integrated architectural approach that describes and visualizes the different architecture domains and their underlying relations and dependencies 16. As for ITIL, it is an English natural language set of documents consisting of several volumes of IT management concepts, processes and methods 8]. Therefore, in the absence of a formal ITIL graphical language and based on our belief that ITIL can be regarded as part of EA, sharing the same domains, components and relationships, we decided to try to model the ITIL meta-model using the language we had already chosen for EA: ArchiMate.

We want however to emphasize that providing a common meta-model of EA and ITIL is a different issue from using a EA oriented language for representing ITIL. In fact, we are proposing the former, so this work isn't the enterprise architecture of ITIL but the modeling of ITIL inside the organization's EA.

Hence, we started by searching through the five ITIL books for concepts that belonged to each of the EA domains. Having them, we built a mapping of these concepts to ArchiMate's metamodel elements based on ITIL and ArchiMate's own definitions. With this concept mapping, we built the ITIL metamodel using ArchiMate concepts for the whole ITIL 26 processes and 4 functions. 
At the end, although we had answered the Zachman Framework's "What", "Where", "When", "Who" and "How" questions, we still lacked the "Why".

To answer this last one, we had to extend the "what is done by the system" approach with the "why is the system like this". This why question is answered in terms of organizational objectives and their impact on information systems supporting the organization [23]. Thus, we chose ArchiMate's Motivation Extension which is used to model the motivations, or reasons, that underlie the design or change of some EA. These motivations influence, guide, and constrain the design [16]. Here we followed a similar course of action: we identified ITIL motivational concepts, mapped them to ArchiMate's concepts, and built motivation models for the ITIL 26 processes and 4 functions.

The research for ITIL graphical representations, the concept maps and the description of the method for the models' construction are out of the scope of this article, and were addressed in two other papers, one about ITIL Business Motivation Model [24] and another one that is awaiting publication.

As we have stated, it is quite possible to identify ITIL components and relationships in every EA domain. Thus, if one starts looking at ITIL from this point of view, we begin to realize that by representing and splitting it across EA realms, we can use composition to integrate them by integrating each of its layers. Therefore, our first proposal is: if an organization can be represented by an enterprise architecture, with all its layers, components and relationships, and if that organization has implemented ITIL, then ITIL components and relationships will be a subset (in every layer) of the EA ones.

On the other hand, an architecture model is not just useful to provide insight into the current or future situation; it can also be used to evaluate the transition from 'as is' to 'to be' [3], and there is a strong relationship between implementing a target EA and an ITIL program. These relationships are manifested in terms of People, Process, Business, and Information [26]. Thus, based on our first proposal that ITIL is part of EA, in the sense that if an organization has ITIL, then in every EA layer there will be ITIL elements, then our second proposal is: implementing ITIL on an organization represented by an EA is the same as implementing any other architectural change, so an EA method for the transition from a baseline to a target architecture could be used to implement ITIL.

\section{Demonstration}

To demonstrate how ITIL fits in EA, we needed to present an organization EA model containing ITIL elements. To achieve this, we decided to use ArchiSurance. The ArchiSurance Case Study is a fictitious example developed to illustrate the use of ArchiMate in the context of TOGAF [27. It concerns the insurance company ArchiSurance, which has been formed as the merging of three independent companies. It describes the baseline architecture before the merging and then a number of change scenarios. TOGAF ADM is then used to go from that baseline architecture to a target one with ArchiSurance after the merging. 
Since this is a running example that is widely used across the ArchiMate community 3151625 28 29] and on ArchiMate training courses [27] we thought it would fit our demonstration purposes. Moreover, The Open Group "expects it to evolve over time, and encourages its members to add new aspects and views or create new change scenarios, as long as they are consistent with the original case description and models" [27]. That said, we start by pointing out that our models are indeed consistent with the existing ones, since we don't subtract anything but add ITIL instead. In fact, our baseline architecture is the target of the ArchiSurance example. Our premise is that after the merging, ArchiSurance was facing the same problems that several other organizations face when they decide to use ITIL. Thus, we will use the exact same approach that is used on the ArchiSurance scenarios examples: we will use the TOGAF ADM and ArchiMate to represent an architecture change from a baseline ("as-is") of ArchiSurance (after the merging) to a target ("to-be") architecture with the implementation of ITIL Service Operation.

Therefore, in the Phase A: Architecture Vision we establish an architecture effort and initiate an iteration of the architecture development cycle by setting its scope, constraints, and goals. Some relevant drivers, assessments and goals are shown in Figure 1 (all the figures are simplified versions of some of our models, due to paper size restrictions). Goals are the basis for requirements, so the next viewpoint we developed was the Goal Refinement viewpoint, which allows to model the refinement of goals into more concrete goals, and its refinement into requirements that describe the properties that are needed to realize the goals 27]. Both of these views were based on our earlier ITIL motivation models.

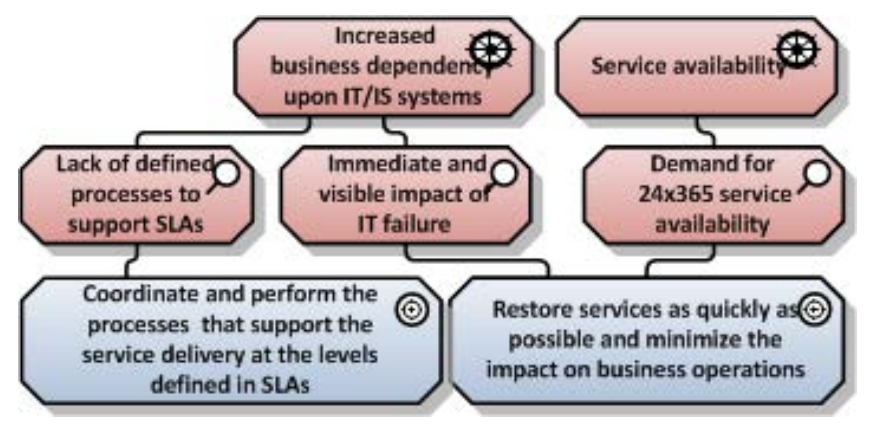

Fig. 1. Detail of Business Goals and Principles

Our next model was the Introductory View, where a simplified notation is used at the start of a design trajectory [27]. Next, we moved on to Phase B: Target Business Architecture and Gap Analysis where we show how the target architecture realizes the key business requirements. For this purpose, TOGAF specifies a Business Footprint diagram. In ArchiMate, this can be expressed using the Requirements Realization viewpoint, which allows the designer to model the realization of requirements by the core elements [27] (Figure 2). 


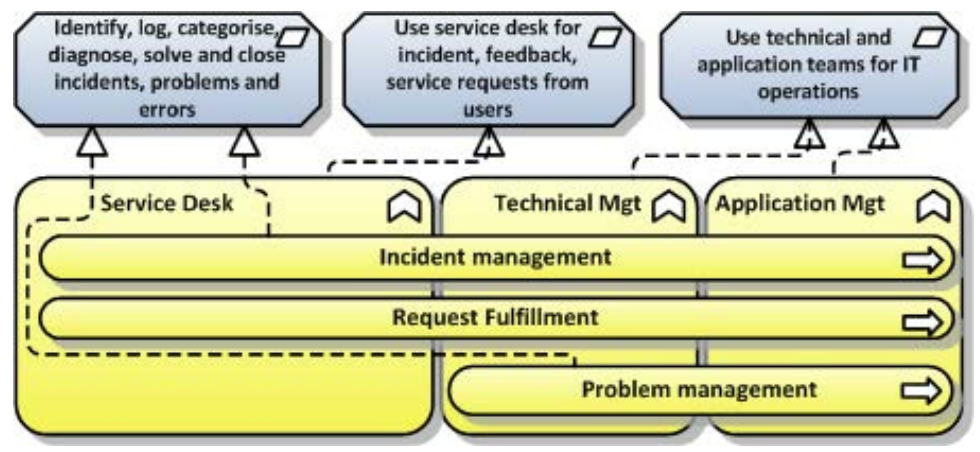

Fig. 2. Detail of Requirements Realization viewpoint

Still on this phase we also show the results of a global gap analysis for the business architecture (Figure 3). In both of these views we used the elements from the business layer of our core ITIL models (in light color), integrating them with ArchiSurance models (in dark color) in this latter view.

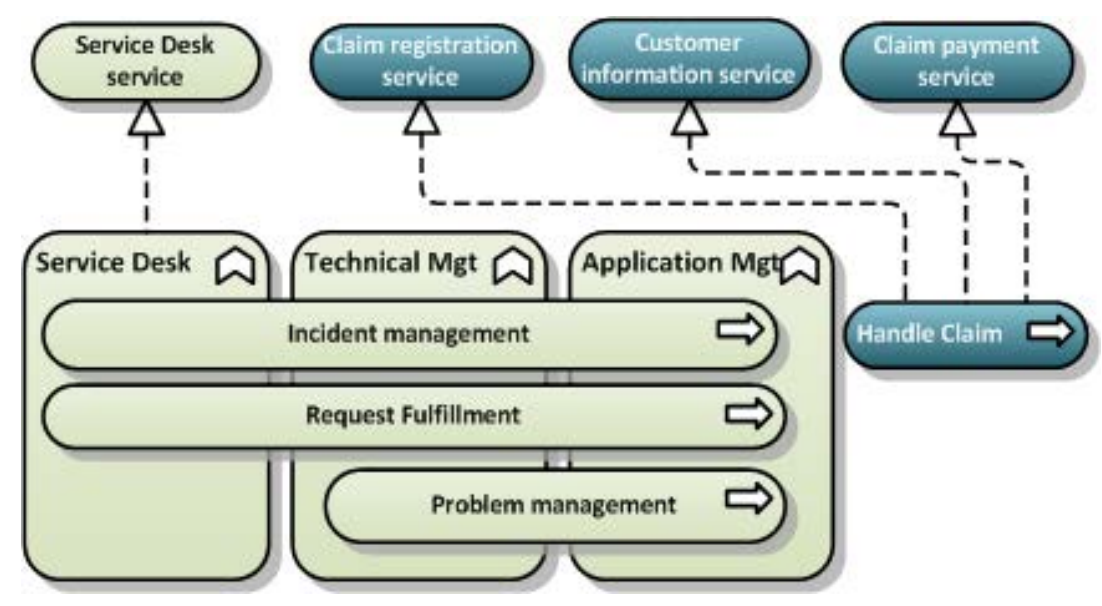

Fig. 3. Detail of target Business Architecture

To formalize the gap analysis we used Rolland's 30] gap typology. Therefore, since we are adding ITIL components to the existing EA, our gaps are mostly "Structural Changes" as they correspond to a modification of the set of elements which composes the model, and are specified by the "AddComponent" operator.

Afterwards, we moved on to Phase C: Target Application Architecture and Gap Analysis, where the Application Communication diagram (Figure 4) shows the proposed target situation for the application landscape, with the results of a global gap analysis for this layer. In the front office, shared service center, and 

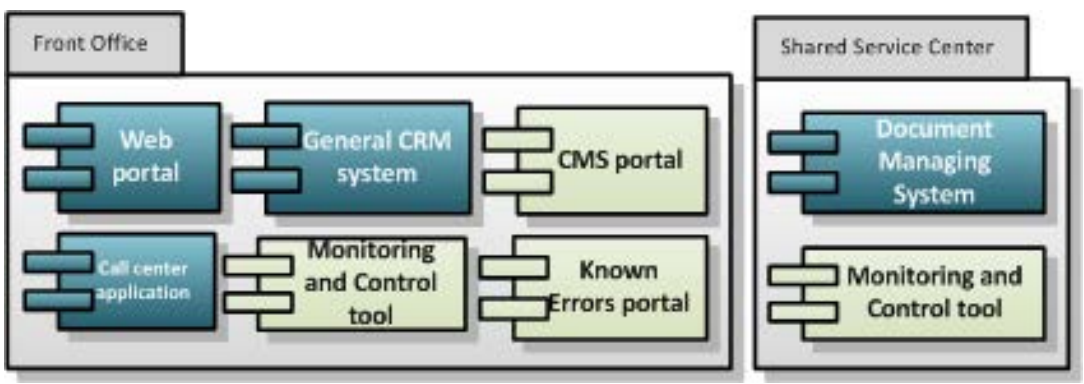

Fig. 4. Detail of target Application Architecture

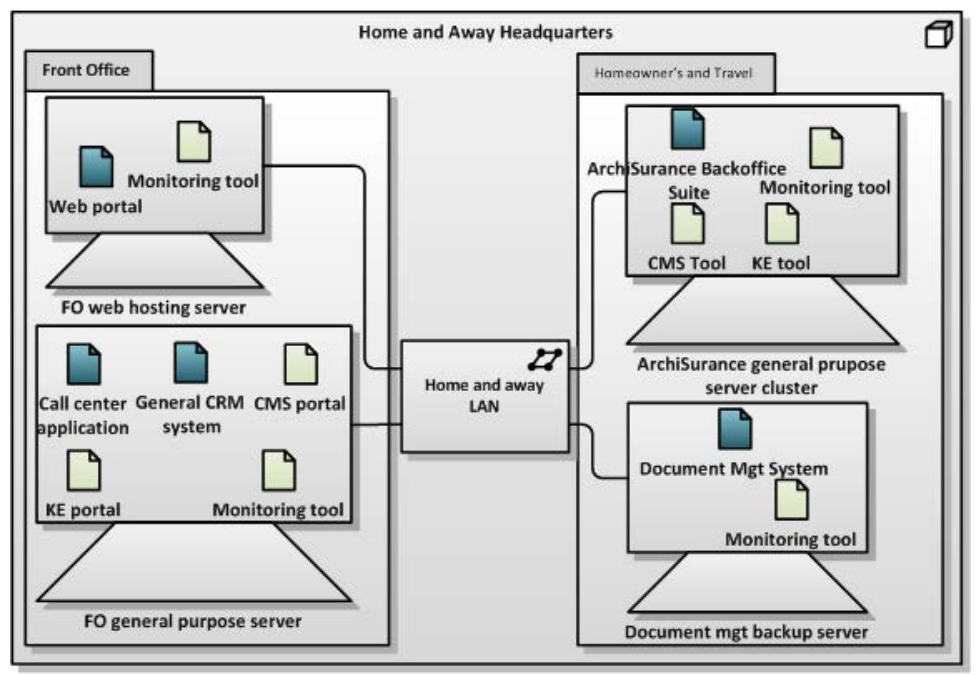

Fig. 5. Detail of target Infrastructure Architecture

back office several ITIL component applications were introduced, like the CMS portal or the Monitoring and Control Tool, with the latter being used to monitor all ArchiSurance baseline applications (in the figure we omitted the relationships for clarity sake). Next, it was time for Phase D: Target Technology Architecture and Gap Analysis, where we use the Infrastructure viewpoint to show the target situation for the infrastructure (Figure 5). Here we introduced ITIL artifacts as the CMS portal or the KE portal which are deployed on the existing (baseline) ArchiSurance infrastructure.

The following step, for Implementation and Migration Planning, TOGAF 9 introduces for Phases $\mathrm{E}$ and $\mathrm{F}$ the transition architecture, representing a possible intermediate situation (plateau) between the baseline and the target architecture. We used ArchiMate's Migration viewpoint to show the baseline, target, and transition architectures, as well as their relationships. Finally, transition 
architectures enable the planning of implementation projects such as Service Desk, Request Fulfillment or Problem Management. The sequence of these projects depends on which of the transition architectures is selected. This can be shown in a TOGAF Project Context which we also developed to link work packages to the functions, services, processes, applications, data, and technology that will be added, removed, or impacted by the project.

Thus, we demonstrated both of our proposals: 1) ArchiSurance is an organization with a EA representation and an ITIL implementation, where the ITIL components (and relationships) are subsets (in every layer) of the EA ones; and 2) we implemented ITIL on an organization represented by an EA, using an EA method as if it was any other architecture change.

\section{Evaluation}

For evaluation we will use the The Moody and Shanks Framework 31. which proposes the following quality factors: completeness, integrity, flexibility, understandability, correctness, simplicity, integration and implementability.

Hence, for completeness we can say our ArchiSurance models contain all user requirements, because these were to implement Service Operation (SO), and our ITIL models contain all the relevant SO elements and relationships. For integrity our models have all the SO rules and constraints, namely the ones that address which are the processes to be implemented, and their application and infrastructure dependencies. They also have flexibility because other ITIL processes can be picked from our overall models and added to ArchiSurance, without changing the model itself. As for understandability the concepts and structures used are ITIL, EA and ArchiMate ones, which are easily recognizable for people in these fields. In fact, and as a side note, when we evaluated our ITIL models through interviews, everyone quickly understood them. For correctness the ArchiSurance "as-is" models are provided by the ArchiMate team themselves, so we can presume correctness, as for our ITIL models, they were built by a method that mapped every ITIL concept to the correct ArchiMate one, followed by its representation according to every ArchiMate rule and convention. The integration with ArchiSurance was also performed according to ArchiMate and TOGAF conventions and rules. We can also find simplicity because in our ArchiSurance models we only show the relevant constructs for a coarse-grained analysis, omitting, for instance, events and business objects from our original ITIL models. Concerning integration, we can see from the final models that the ITIL elements and relationships fit and are consistent with the baseline architecture in every layer. And finally, for implementability we know the implementation is possible, because it is simply the implementation of ITIL Service Operation, something that is done quite often in organizations.

\section{Conclusion}

With this work, we tried to close this gap between EA and ITIL, and propose an integration through a common frame of reference, a graphical modeling 
language. In fact, like ArchiMate's own goal itself, our objective was also "to provide domain integration through an architecture language and visualization techniques that picture these domains and their relations, providing the architect with instruments that support and improve the architecture process" [16.

The practical outcome of our approach is that once one start thinking about implementing ITIL as an architecture change, one can use architecture tools and methods to perform it. With our ITIL models, an architect has now the tools to design ITSM organizations, hand-picking from our motivation models his organization's specific motivations and tracing them to the right ITIL processes to implement. He can model both the baseline and target architecture with our ITIL components, and then, through gap analysis on each EA layer, he will be able to see which people, information, processes, tools or infrastructure they will need to buy, keep, develop or change in order to reach the intended ITIL target.

On the other hand, this paper also wants to start a discussion about using EA for best practices in specific domains, which can be ITSM, but could as well be purchasing or logistics. In fact, by defining the motivations and architecture in each domain, one can design specific organizations and evaluate and assert their best practices' compliance.

In short, in times where cost and value generation are such important drivers, IT governance, more than ever, should turn organizations more effective and efficient. Therefore, we hope this contribution can help to better understand EA and ITIL, two worldwide standards, complementary on organizations, with distinct IT and organizational perspectives, yet so close that they have much more to gain from aligning together instead of walking apart.

\section{References}

1. Henderson, J.C., Venkatraman, N.: Strategic alignment: Leveraging information technology for transforming organizations. IBM Systems Journal 32, 472-484 (1993)

2. Gama, N., Sousa, P., Mira da Silva, M.: Integrating Enterprise Architecture and IT Service Management. In: 21st International Conference on Information Systems Development (ISD 2012) (2012)

3. Lankhorst, M., et al.: Enterprise Architecture at Work. Springer, Berlin (2009)

4. The Open Group: The Open Group Architecture Framework. Version 9 (2011)

5. Zachman, J.: A Framework for Information Systems Architecture. IBM Systems Journal 26, 276-292 (1987)

6. The Stationery Office: The Official Introduction to ITIL Service Lifecycle (2007)

7. Hanna, A., Windebank, J., Adams, S., Sowerby, J., Rance, S., Cartlidge, A.: ITIL V3 Foundation Handbook. The Stationary Office, Norwich (2008)

8. Hochstein, A., Zarnekow, R., Brenner, W.: ITIL as common practice reference model for IT service management: formal assessment and implications for practice. In: IEEE International Conference on eTechnology eCommerce, vol. 21, pp. $704-710(2005)$

9. Arraj, V.: ITIL: The Basics White Paper. The Stationary Office (2010)

10. Hevner, A.R., March, S.T., Park, J., Ram, S.: Design Science in Information Systems Research. MIS Quarterly 28, 78-105 (2004) 
11. Greefhorst, D., Proper, E.: Architecture Principles: The Cornerstones of Enterprise Architecture. Springer, Berlin (2011)

12. Ross, J.W., Weill, P., Robertson, D.C. (eds.): Enterprise Architecture As Strategy: Creating a Foundation for Business Execution. Harvard Business Scholl Press, Boston (2006)

13. Pereira, C., Sousa, P.: A Method to Define an Enterprise Architecture using the Zachman Framework. In: The 19th Annual ACM Symposium on Applied Computing (SAC 2004), Nicosia, Cyprus, pp. 1366-1371. ACM (2004)

14. Van Sante T., Ermersj J.: TOGAF 9 and ITIL V3. White Paper (2009)

15. Jonkers, H., Proper, E., Turner, M.: TOGAF 9 and ArchiMate 1.0. White Paper. The Open Group (2009)

16. The Open Group, Archimate 2.0 Specification. The Open Group (2012)

17. Jan van Bon, et al.: Foundations of IT Service Management Based on ITIL v3. Van Haren Publishing (2007)

18. Braun, C., Winter, R.: Integration of IT Service Management Into Enterprise Architecture. In: ACM Symposium on Applied Computing, pp. 1215-1219. ACM, New York (2007)

19. Nabiollahi, A., Alias, R.A., Sahibuddin, S.: A Service Based Framework for Integration of ITIL V3 and Enterprise Architecture. In: 2010 International Symposium in Information Technology (ITSim), Kuala Lumpur, vol. 1, pp. 1-5 (2010)

20. Taylor, S., Lloyd, V., Rudd, C. (eds.): ITIL: Service Design. TSO, Norwich (2007b)

21. Thorn, S.: TOGAF and ITIL. Catalog number W071, p. 26. The Open Group, San Francisco (2007)

22. Correia, A., Abreu, F.B.: Integrating IT Service Management within the Enterprise Architecture. In: 4th ICSEA, Porto, Portugal, pp. 553-558 (2009)

23. Rolland, C.: Capturing System Intentionality with Maps. In: Krogstie, J., Opdahl, A.L., Brinkkemper, S. (eds.) Conceptual Modelling in Information Systems Engineering, pp. 141-158. Springer (2007)

24. Vicente, M., Gama, N., Mira da Silva, M.: Modeling ITIL Business Motivation Model in ArchiMate. In: Falcão e Cunha, J., Snene, M., Nóvoa, H. (eds.) IESS 2013. LNBIP, vol. 143, pp. 86-99. Springer, Heidelberg (2013)

25. Lankhorst M. and the ArchiMate team: ArchiMate Language Primer (2004)

26. Radhakrishnan, R.: Enterprise Architecture \& IT Service Management - ITSM Frameworks and Processes and their Relationship to EA Frameworks and Processes. The Open Group (2008)

27. Jonkers, H., Band, I., Quartel, D.: ArchiSurance Case Study. The Open Group (2012)

28. Lankhorst, M., Drunen, H.: Enterprise Architecture Development and Modelling. Information Systems Journal 8, 1-16 (2007), Via Nova Architectura

29. Meertens, L.O., Iacob, M.E., Nieuwenhuis, L.J.M., van Sinderen, M.J., Jonkers, H., Quartel, D.: Mapping the business model canvas to ArchiMate. In: Proceedings of the 27th Annual ACM Symposium on Applied Computing, pp. 1694-1701. ACM (2012)

30. Rolland, C., Salinesi, C., Etien, A.: Eliciting gaps in requirements change, Requirements Engineering, vol. 9, p. 115. ST Eliciting gaps in requirements change (2004)

31. Moody, D.L., Shanks, G.G.: Improving the Quality of Data Models: Empirical Validation of a Quality Management Framework. Information Systems 28, 619-650 (2003) 